\title{
Detecting and Identifying Industrial Gases by a Method Based on Olfactory Machine at Different Concentrations
}

\author{
Yunlong Sun (D), ${ }^{1}$ Dehan Luo, ${ }^{1}$ Hui Li, ${ }^{1}$ Chuchu Zhu, \\ Ou Xu, ${ }^{1}$ and Hamid Gholam Hosseini ${ }^{2}$ \\ ${ }^{1}$ School of Information and Engineering, Guangdong University of Technology, Guangzhou 510006, China \\ ${ }^{2}$ Department of Electrical and Electronic Engineering, Auckland University of Technology, Auckland, New Zealand \\ Correspondence should be addressed to Yunlong Sun; sunous1989@163.com
}

Received 16 August 2017; Accepted 1 January 2018; Published 1 March 2018

Academic Editor: Ephraim Suhir

Copyright (C) 2018 Yunlong Sun et al. This is an open access article distributed under the Creative Commons Attribution License, which permits unrestricted use, distribution, and reproduction in any medium, provided the original work is properly cited.

\begin{abstract}
Gas sensors have been widely reported for industrial gas detection and monitoring. However, the rapid detection and identification of industrial gases are still a challenge. In this work, we measure four typical industrial gases including $\mathrm{CO}_{2}, \mathrm{CH}_{4}, \mathrm{NH}_{3}$, and volatile organic compounds (VOCs) based on electronic nose (EN) at different concentrations. To solve the problem of effective classification and identification of different industrial gases, we propose an algorithm based on the selective local linear embedding (SLLE) to reduce the dimensionality and extract the features of high-dimensional data. Combining the Euclidean distance (ED) formula with the proposed algorithm, we can achieve better classification and identification of four kinds of gases. We compared the classification and recognition results of classical principal component analysis (PCA), linear discriminate analysis (LDA), and PCA + LDA algorithms with the proposed SLLE algorithm after selecting the original data and performing feature extraction. The experimental results show that the recognition accuracy rate of the SLLE reaches $91.36 \%$, which is better than the other three algorithms. In addition, the SLLE algorithm provides more efficient and accurate responses to high-dimensional industrial gas data. It can be used in real-time industrial gas detection and monitoring combined with gas sensor networks.
\end{abstract}

\section{Introduction}

Industrial gases are widely produced in industrial raw materials manufacturing and result in environmental pollution and disastrous accidents. Therefore, industrial gases need to be rapidly and accurately detected and identified. It is a valuable work. Electronic nose (EN) has been rapidly developed as a novel gas detection technology in the past two decades. It is widely implicated in toxic/harmful gas detection [1], food safety [2], environmental monitoring [3], medical industry [4], and other fields [5-10].

Sensors and pattern recognition methods are hot topics in the field of electronic nose (EN) systems and identification tools. Hossein-Babaei and Hooshyar Zare reported a novel conductive polymer electrochemical sensor for the detection of hexane, benzene, and CO [11].

On the other hand, the pretreatment of odor detection signal, feature extraction, and feature space reconstruction have attracted more and more attention. A common tin dioxide gas sensor to identify complex odors was reported [12], which was dependent on the heating function of the device with a predetermined temperature that affected the voltage pulses to produce different odor signal differences.

Furthermore, the classical linear dimensionality methods such as the principal component analysis (PCA) and the Fisher linear discriminate analysis (FLDA) combined with the extreme learning machine (ELM) were applied to the determination of Chinese liquor quality [13] and fruit freshness [14].

The learning method of artificial neural networks (ANN) has a good effect applied to detection of cigarettes and tea quality and freshness of chicken [15-17]. The electronic nose (EN) system was also used in the diagnosis of respiratory diseases [18] and rapid identification of Chinese herbal medicines [19], and the machine olfactory joint robot is applied to the collection of local odor source positioning [20]. However, the current electronic nose detection and identification of the report mainly focused on qualitative analysis of the material. 


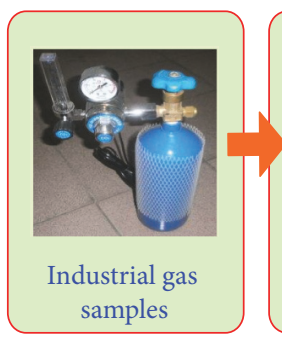

(a)

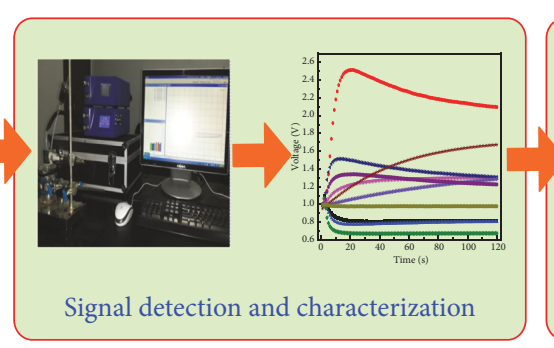

(b)

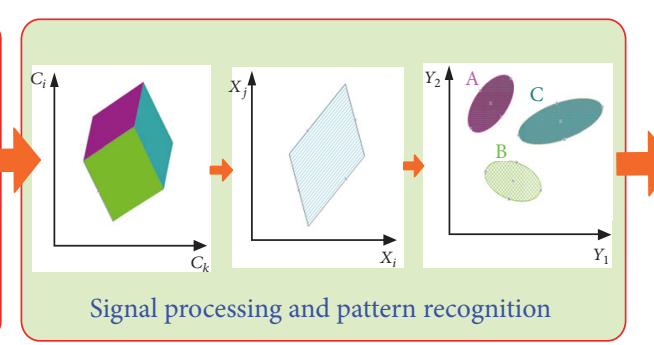

(c)

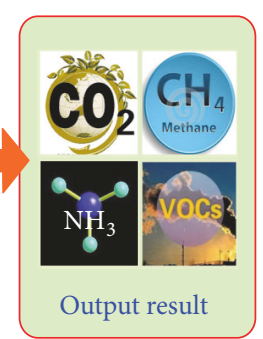

(d)

FIGURE 1: Industrial gas detection and identification of experimental system. (a) Gas samples storage and flow control. (b) The gas signal is detected and stored using the electronic nose (EN) system. (c) Then the pattern recognition method is used to process and identify the gas signal (c). (d) Finally the recognition result is output.

TABLE 1: Samples and experimental set-up.

\begin{tabular}{lcccc}
\hline Gas type & NH3 & CO2 & CH4 & VOCs \\
\hline & 150 & 150 & 150 & 150 \\
Concentration & 300 & 300 & 300 & 300 \\
(ppm) & 450 & 450 & 450 & 450 \\
& 600 & 600 & 600 & 600 \\
\hline Temperature & \multicolumn{5}{c}{$22 \pm 1^{\circ} \mathrm{C}$} \\
\hline Relative & $60 \pm 10 \%$ \\
humidity & \multicolumn{5}{c}{$120 \mathrm{~S}$} \\
\hline Sampling time
\end{tabular}

How to detect and identify the same substance at different concentrations is a higher challenge.

At the same time, when multiple sensor arrays of the electronic nose system are used, the acquired high-dimensional raw data is the reason of "dimensional disaster." In this study, we propose the selective local linear embedding (SLLE) method to solve the problem of dimensionality reduction and classification of high-dimensional data in industrial gas detection. Comparing with the classification and recognition results of classical PCA, LDA, and PCA + LDA, the experimental results visually demonstrate the features and benefits of the SLLE algorithm. This provides a new method for rapid detection and identification of industrial gases.

\section{Experimental and Methods}

2.1. Experimental System of Electronic Nose. Industrial gas detection experimental structure is shown in Figure 1. Firstly, the industrial gas sample is stored in the reinforced steel bottle, and the gas releases the gas flow by adjusting the air pressure valve. Then, when the gas flows in the electronic nose sampling interface, the sensor array responds and records the gas response map. The saved response map data will be used for further feature extraction and analysis by the pattern recognition method and finally outputting the identified results.

We used a dynamic cylinder formulation method for standard gas distribution, by controlling the flow rate and time of the release gas, mixed with pure dry air. The samples gas is shown in Table 1.
In this study, we used the PEN3 electronic nose (Germany, AIRSENSE Ltd.) to measure different concentrations of industrial gases. The PEN3 consists of 10 metal oxide sensor arrays, as shown in Table 2.

The electronic nose technique simulated the olfactory function of organism, transformed the multidimensional response signal into the sensory evaluation index value, and completed the intelligent explanation of the qualitative and quantitative analysis results of odor.

The detailed information of the selected electronic nose and its technical specifications is presented in Supplementary Materials Table S1.

We measured the gas samples at room temperature and other room conditions (the temperature of $22 \pm 1^{\circ} \mathrm{C}$, relative humidity of $60 \pm 10 \%$ ). The sampling period is 120 seconds and the intake air flows when sampling is set to $7.76 \mathrm{ml} / \mathrm{s}$.

2.2. Sensor Acquisition Signal. We obtained a typical four gas response map and a response to the sensor array when the concentration was $300 \mathrm{ppm}$ via the electronic nose (EN), as shown in Figure 2.

This diagram clearly shows the response values of the 10 sensors for different signal intensities of carbon dioxide $\left(\mathrm{CO}_{2}\right)$, methane $\left(\mathrm{CH}_{4}\right)$, ammonia $\left(\mathrm{NH}_{3}\right)$, and volatile organic compounds (VOCs) at different time periods. We can find that the response values between 50 and 100 seconds show significant differences between the four gases in the response map. It can extract odor information effectively and distinguish four kinds of gases. Therefore, using electronic nose system to build odor libraries of different gases can help to identify multiphase gases and identify complex gases.

\subsection{Data Processing Method}

2.3.1. An Overview of PCA. Principal component analysis (PCA) is one of the widely used linear dimensionality reduction methods. The key to the kernel is to measure the size of the variance as a measurement of the amount of information that the greater the variance, the more information provided, and vice versa. PCA through the original component of the linear combination of large variance have a large amount of information on the main components, thereby reducing the data dimension. The calculation process of PCA can be realized by matrix singular value decomposition (SVD). 
TABLE 2: Electronic nose sensors parameters of AIRSENSE PEN3.

\begin{tabular}{lccc}
\hline Sensor number & Sensor name & Object substances for sensing & Limit of detection \\
\hline Sensor 1 & W1C & Aromatics & $5 \mathrm{ppm}$ \\
Sensor 2 & W5S & Ammonia and aromatic molecules & $1 \mathrm{ppm}$ \\
Sensor 3 & W3C & Broad-nitrogen oxide & $5 \mathrm{ppm}$ \\
Sensor 4 & W6S & Hydrogen & $5 \mathrm{ppm}$ \\
Sensor 5 & W5C & Methane, propane, and aliphatics & $1 \mathrm{ppm}$ \\
Sensor 6 & W1S & Broad-methane & $5 \mathrm{ppm}$ \\
Sensor 7 & W1W & Sulfur-containing organics & $0.1 \mathrm{ppm}$ \\
Sensor 8 & W2S & Broad-alcohols, broad-carbon chains & $5 \mathrm{ppm}$ \\
Sensor 9 & W2W & Aromatics, sulfur- and chlorine-containing organics & $1 \mathrm{ppm}$ \\
Sensor 10 & W3S & Methane and aliphatics & $5 \mathrm{ppm}$ \\
\hline
\end{tabular}

2.3.2. An Overview of LDA. Linear discriminant analysis (LDA) is a generalization of Fisher's linear discriminant, a method used in statistics, pattern recognition, and machine learning to find a linear combination of features that characterizes or separates two or more classes of objects or events. The resulting combination may be used as a linear classifier or, more commonly, for dimensionality reduction before the classification.

2.3.3. Selective Local Linear Embedding (SLLE) Method. Local linear embedding (LLE) is a very important dimensionality reduction method. Compared with traditional principal component analysis (PCA) and linear discriminant analysis (LDA), the LLE focuses on the linear features of the local area of the sample when the dimensionality is reduced. It is mainly through complex nonlinear region, divided into multiple small linear local areas. Then by dimensionality reduction, the dimensionality-preserving data contains more linear features of the original data. However, the LLE algorithm has some shortcomings. For example, the LLE algorithm is limited by the unevenness of the training samples and affects the distribution of the feature space after dimensionality reduction.

In this study, selective local linear embedding (SLLE) was proposed to reconstruct a linear combination of neighborhoods in a sample region. Each sample point area can be reconstructed by a linear combination of multiple neighbor points. When the dimensionality reduction $K$ of the output is selected, the optimal dimensionality reduction feature space is calculated by calculating the minimum reconstruction error and the weight of the reconstruction sample point in the low-dimensional space, keeping the weight of the local neighborhood unchanged and distributed. The SLLE algorithm and its steps are described in Figure 7.

In Step 1, the space distance between each sample point and other sample points is calculated, and the nearest $\mathbf{k}$ sample point is used as the nearest neighbor of the sample point. The distance formula is as follows:

$$
d_{i j}=\sqrt{\sum_{k=1}^{D}\left|x_{i k}-x_{j k}\right|^{2}}
$$

In Step 2, the error function $\mathbf{W}$ is defined as follows:

$$
\min \varepsilon(W)=\sum_{i=1}^{N}\left|x_{i}-\sum_{j=1}^{k} w_{i j} x_{i j}\right|^{2}
$$

where $x_{i j}(j=1,2, \ldots, k)$ is the $\mathbf{K}$ neighbors of $x_{i} \cdot w_{i j}$ denotes the weighting coefficient of the $\mathrm{jth}$ nearest neighbor when reconstructing $\mathbf{X}_{\mathbf{i}}$ from the linear combination of $\mathbf{k}$ nearest neighbors satisfying the following equation:

$$
\sum_{j=1}^{k} w_{i j}=1
$$

Then (2) can be equivalent to

$$
\begin{gathered}
\min \quad \\
\varepsilon(W)=\sum_{i=1}^{N}\left|x_{i}-\sum_{j=1}^{k} w_{i j} x_{i j}\right|^{2}=\sum_{i=1}^{N}\left|x_{i} \sum_{j=1}^{k} w_{i j}-\sum_{j=1}^{k} w_{i j} x_{i j}\right|^{2}=\sum_{i=1}^{N}\left|\sum_{j=1}^{k} w_{i j}\left(x_{i}-x_{i j}\right)\right|^{2} \\
=\sum_{i=1}^{N}\left|\left[\left(x_{i}-x_{i 1}\right),\left(x_{i}-x_{i 2}\right), \ldots,\left(x_{i}-x_{i k}\right)\right]\left[w_{i 1}, w_{i 2}, \ldots, w_{i k}\right]^{T}\right|^{2} .
\end{gathered}
$$

Let $W_{i}=\left[w_{i 1}, w_{i 2}, \ldots, w_{i k}\right]^{T}$; it represents the local reconstruction weight vector for the ith sample point. Then

$$
\min \varepsilon(W)=\sum_{i=1}^{N}\left[\left(x_{i}-x_{i 1}\right),\left(x_{i}-x_{i 2}\right), \ldots,\left(x_{i}-x_{i k}\right) W_{i}\right]^{T}\left[\left(x_{i}-x_{i 1}\right),\left(x_{i}-x_{i 2}\right), \ldots,\left(x_{i}-x_{i k}\right) W_{i}\right]
$$




$$
=\sum_{i=1}^{N} W_{i}^{T}\left[\left(x_{i}-x_{i 1}\right),\left(x_{i}-x_{i 2}\right), \ldots,\left(x_{i}-x_{i k}\right)\right]^{T}\left[\left(x_{i}-x_{i 1}\right),\left(x_{i}-x_{i 2}\right), \ldots,\left(x_{i}-x_{i k}\right)\right] W_{i}
$$

Let $M_{i}=\left[\left(x_{i}-x_{i 1}\right),\left(x_{i}-x_{i 2}\right), \ldots,\left(x_{i}-x_{i k}\right)\right]^{T}\left[\left(x_{i}-\right.\right.$ $\left.\left.x_{i 1}\right),\left(x_{i}-x_{i 2}\right), \ldots,\left(x_{i}-x_{i k}\right)\right]$, and then

$$
\min \varepsilon(W)=\sum_{i=1}^{N} W_{i}^{T} M_{i} W_{i} .
$$

Equation (6) can be solved by LaGrange multiplier method as

$$
L(W)=\sum_{i=1}^{N}\left[W_{i}^{T} M_{i} W_{i}+\lambda\left(\sum_{j=1}^{k} w_{i j}-1\right)\right]
$$

Then by taking the partial derivative of $W_{i}$ on both sides of (7) at the same time we can solve for $W_{i}$ :

$$
\begin{aligned}
\frac{\partial L(W)}{\partial W_{i}} & =2 M_{i} W_{i}+\lambda=0 \\
W_{i} & =-\frac{\lambda}{2} M_{i}^{-1}
\end{aligned}
$$

In Step 3, the output dimension matrix $\mathbf{Y}$ is calculated. The matrix $Y$ should satisfy the following conditions:

$$
\begin{gathered}
\min \psi(Y)=\sum_{i=1}^{N}\left|Y_{i}-\sum_{j=1}^{k} W_{i} Y_{i j}\right|^{2} . \\
\sum_{i=1}^{N} Y_{i}=0 \\
\frac{1}{N} \sum_{i=1}^{N} Y_{i} Y_{i}^{T}=I
\end{gathered}
$$

Then,

$$
\begin{aligned}
\min & \psi(Y)=\sum_{i=1}^{N}\left|Y_{i}-\sum_{j=1}^{k} W_{i} Y_{i j}\right|^{2}=\sum_{i=1}^{N}\left|Y I_{i}-Y W_{i}\right|^{2} \\
& =|Y(I-W)|^{2}=[Y(I-W)][Y(I-W)]^{T} \\
& =Y(I-W)(I-W)^{T} Y^{T} .
\end{aligned}
$$

Let $P=(I-W)(I-W)^{T}$; then $\min \psi(Y)=Y P Y^{T}$.

Repeat Step 2 of the solution process, available:

$$
Y_{i}=-\lambda P_{i}^{-1}
$$

Finally, we select the eigenvalue of the smallest $\mathbf{k}$ characteristic values of the matrix $\mathbf{P}$ as the dimensionality reduction output. In order to minimize the loss function value, the eigenvalues of the reconstructed matrix $\mathbf{P}$ are calculated. The eigenvalues from the 2 nd to the $\mathbf{k}+\mathbf{1}$ th index are selected, as well as the corresponding eigenvector as the output of the dimensionality reduction.
2.3.4. Euclidean Distance (ED) Formula. In this study, the Euclidean distance (ED) formula was used for classifying the testing samples. The calculation was based on the twodimensional sample feature space using the following formula:

$$
\mathbf{d}=\sqrt{\left(x_{\text {test } 1}-x_{\text {train } 2}\right)^{2}+\left(y_{\text {test } 1}-y_{\text {train } 2}\right)^{2}} .
$$

The absolute distance value $\mathbf{d}$ is an effective reference for discriminating the classification, where $x_{\text {test } 1}$ and $y_{\text {train } 1}$ are the two-dimensional feature distributions of the testing samples; $x_{\text {test } 2}$ and $y_{\text {train } 2}$ are the two-dimensional feature distributions of the training samples.

\section{Results and Discussion}

In order to show the characteristics of the proposed method in a concise and intuitive manner, we select a total of 124 samples (31 groups of each class) of the four measured gases to train the classification. The gas concentration can reach 300 ppm, through the electronic nose measurement. Firstly, we present a detailed classification of four types of sample gas training results, through PCA, LDA, and PCA + LDA before and after feature extraction. Finally, the results of SLLE algorithm training classification presented in this study are compared with the results shown before.

3.1. Classification Results of Previous Feature Extraction. The classifications results of steady-state PCA, steady-state LDA, and steady-state PCA + LDA before the feature extractions are shown in Figures 3(a), 3(b), and 3(c), respectively. We can find that the four gas samples in the graph can not be clearly classified based on the original data. Based on sensor variance analysis, many data points overlap in twodimensional distribution space.

3.2. Classification Results after Feature Extraction. The classification results show three classification methods after feature extraction in Figure 4. In Figure 4(a), the classification results of the four gas samples are shown. Black, red, green, and blue cross represent $\mathrm{CO}_{2}, \mathrm{CH}_{4}, \mathrm{NH}_{3}$, and VOCs, respectively. Since the degree of polymerization within the class is not good, the classification of the sample is scattered in the space, and even the samples are wrongly divided. Thus, PCA is not the optimal method for this study.

It clearly shows the classification of four gases through LDA, in Figure 4(b). Simultaneously, we can find that the classification effect is significantly better than PCA. However, due to the limited projection space, we can find that when the sample data are highly similar, the interclass distance of the sample classification is not obvious and prone to misjudgment. 


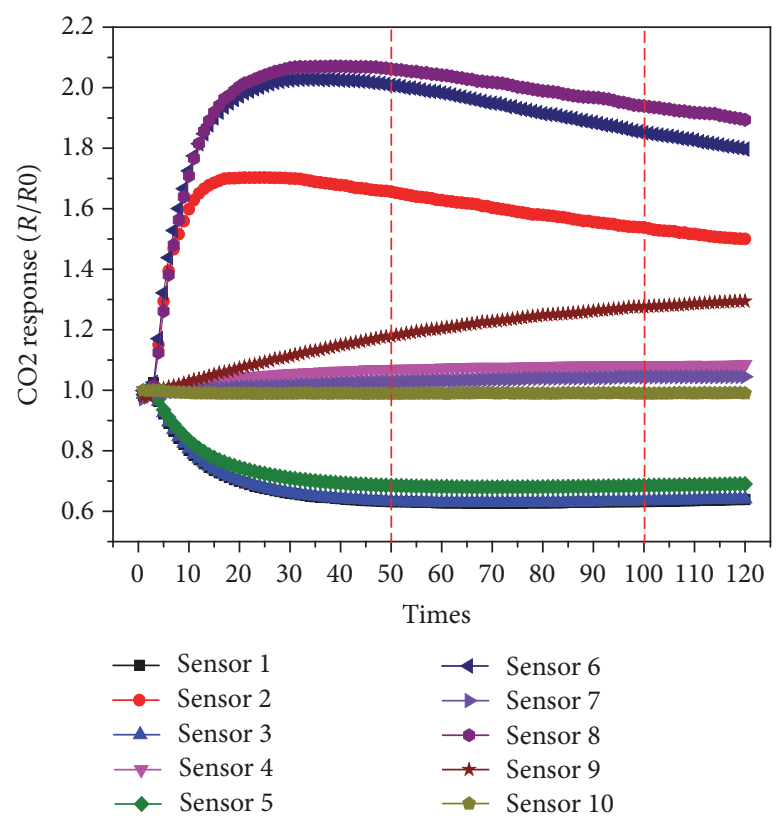

(a)

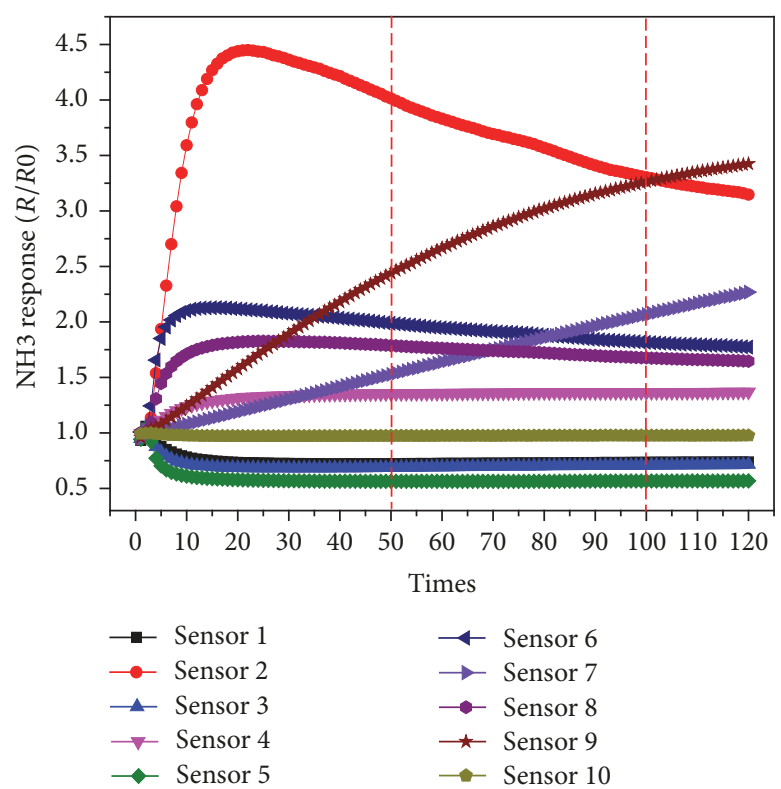

(c)

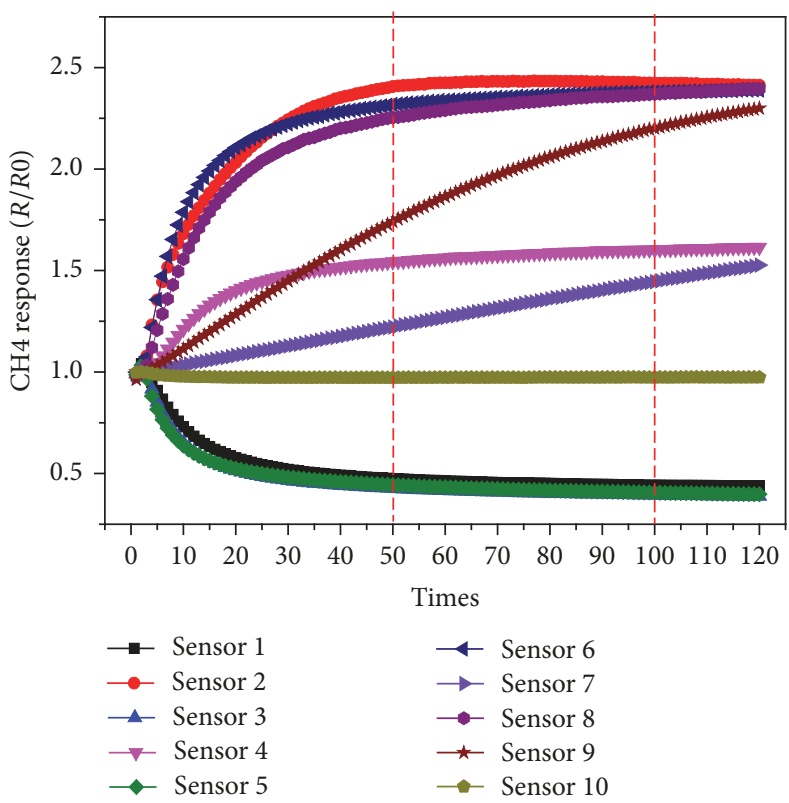

(b)

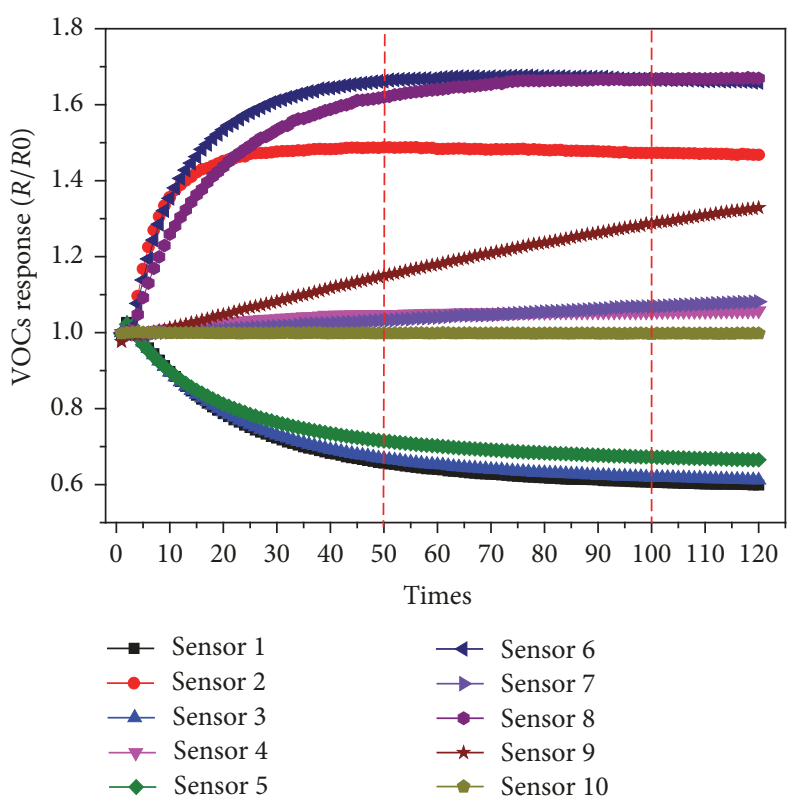

(d)

Figure 2: The sensor response map of $\mathrm{CO}_{2}, \mathrm{CH}_{4}, \mathrm{NH}_{3}$, and $\mathrm{VOC}_{\mathrm{S}}$ corresponds to (a), (b), (c), and (d), respectively, when the concentration was $300 \mathrm{ppm}$. The horizontal axis stands for the sampling time $(0-120 \mathrm{~s})$ and the vertical axis denotes the amplitude of sensor output impedance ratio.

In Figure 4(c), the classification results are processed by PCA + LDA. PCA is used for the first step of the data to be dimensionally reduced, and then the reduced dimension data is calculated by optimizing the classification projection space through LDA. However, due to the high overlap and similarity of the original data samples, we can find that the classification space of the four gas samples has improved but is not optimal from the classification results.
3.3. Classification Results of SLLE. The results of the reduction and classification of the four industrial gases by the SLLE algorithm are shown in Figure 5.

Figures 5(a), 5(b), and 5(c) correspond to the classification results, when the selected sample nearest neighbor number $K=10,20,30$, respectively. We can find that the spatial distribution of the four gas samples is significantly better than that of Figures 3 and 4. Because SLLE algorithm is 


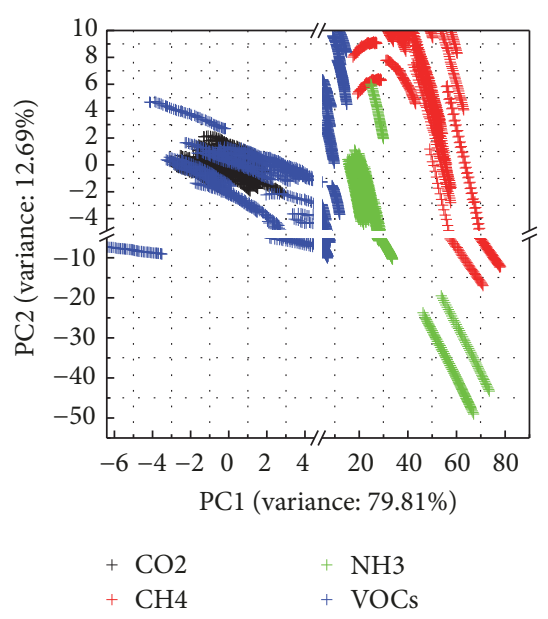

(a)

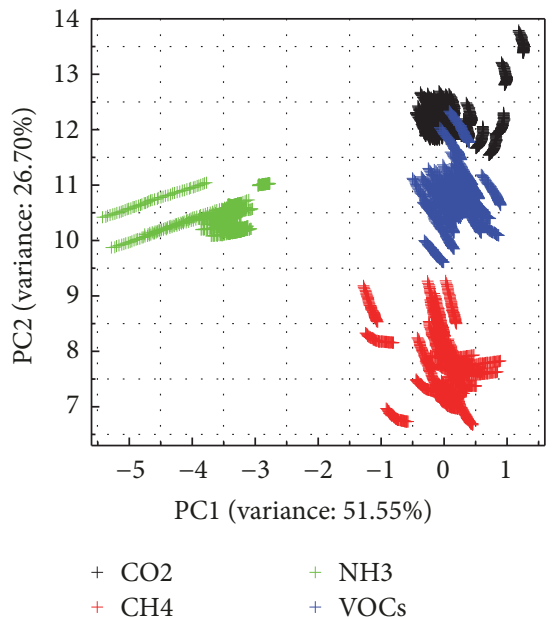

(b)

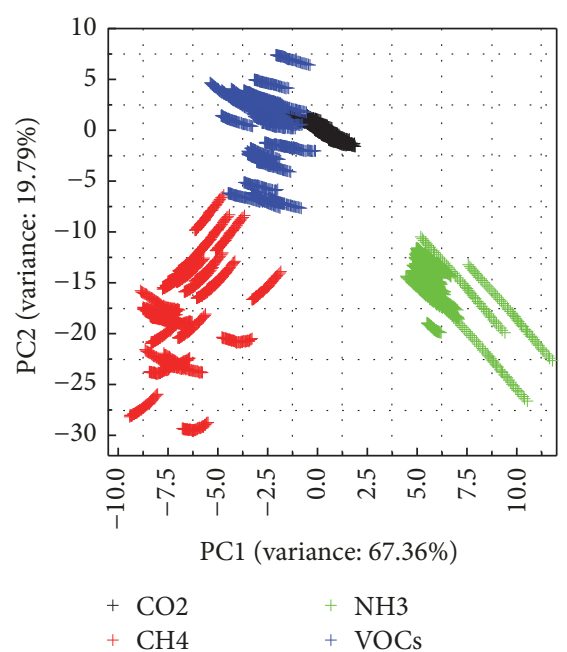

(c)

FIgURE 3: Classification results of steady-state PCA, steady-state LDA, and steady-state PCA + LDA method before the feature extraction corresponding to (a), (b), and (c), respectively. The horizontal axis represents the proportion of the first variance (eigenvalues corresponding to the eigenvalues), and the vertical axis represents the proportion of the second variance, and the two constitute the original classification in the two-dimensional distribution space.

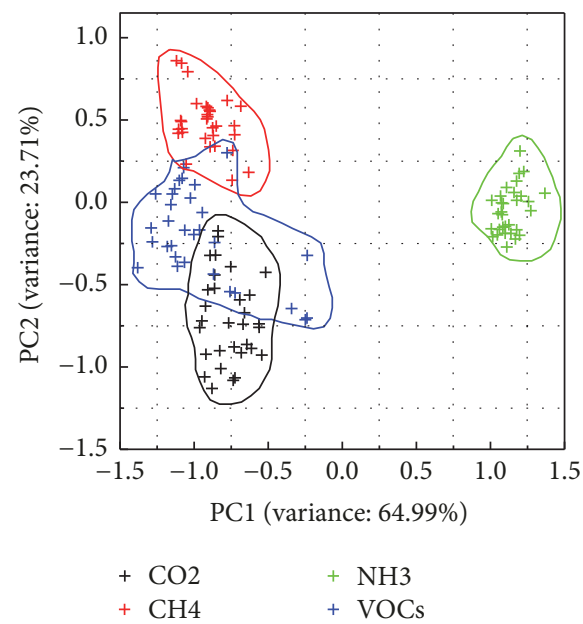

(a)

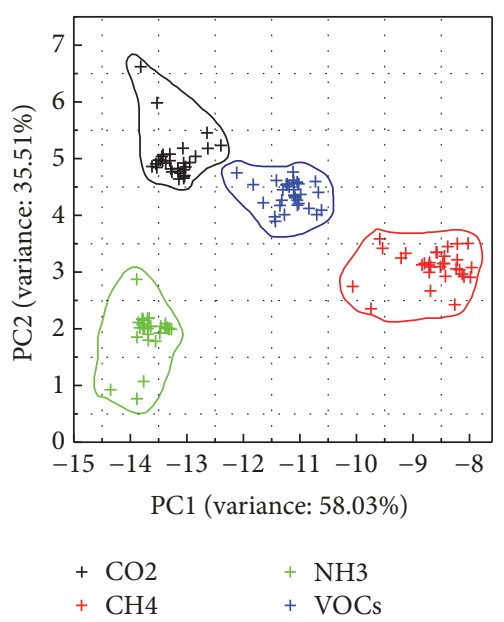

(b)

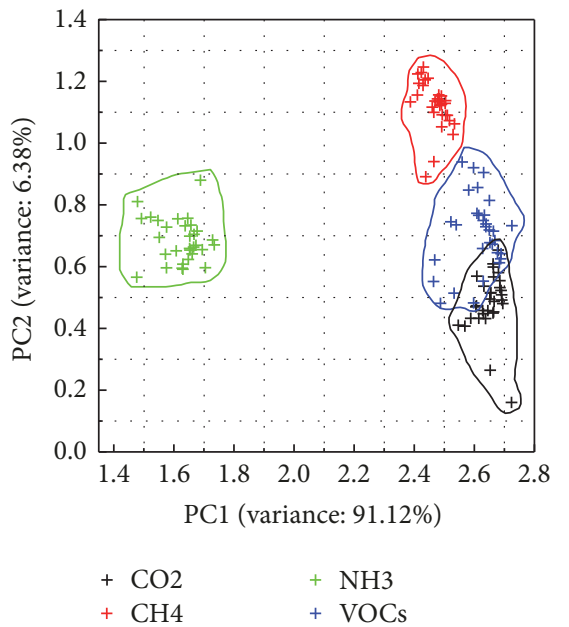

(c)

Figure 4: Classification results of PCA, LDA, and PCA + LDA method after the feature extraction corresponding to (a), (b), and (c), respectively. The horizontal axis and the vertical axis stand for the dimensionally reduced classification in the two-dimensional distribution space.

characterized by the linear dimensionality reduction of highdimensional complex data, it can maintain its spatial features and facilitate feature reconstruction.

Furthermore, we can classify high-dimensional, nonlinear, and high-similar sample data by selecting the neighborhood of the sample and the dimension of the output sample. However, its flaws are also significant. As the nearest neighbor of the selected sample space increases, its computational complexity is also increased, and the computational complexity is $O\left(N^{2}\right)$ (equivalent to $N * N$ matrix calculation).
3.4. Comparison of Recognition Rate of Four Kinds of Algorithms. The classification training for four sample gases' data has been presented in Section 3. The test recognition results parameters are shown in Table 3.

In Table 3, the data sets of four gas samples of 168 groups, 124 groups, 95 groups, and 84 groups were trained, and 115 groups, 80 groups, 64 groups, and 81 groups were tested, respectively.

The accuracy of the four gas samples is shown in Figure 6. We can find four samples' test accuracy from $73.43 \%$ to $91.36 \%$. In detail, the accuracy of the SLLE test is close to $90 \%$, 


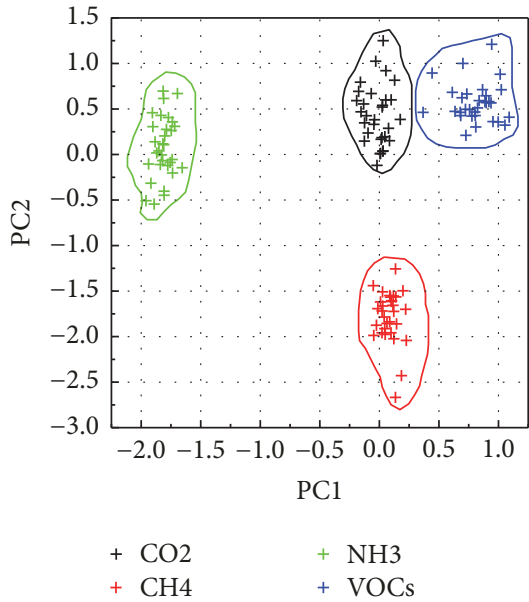

(a)

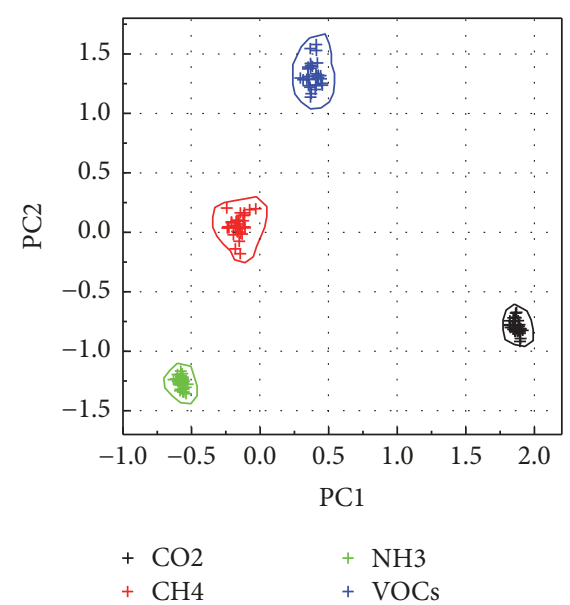

(b)

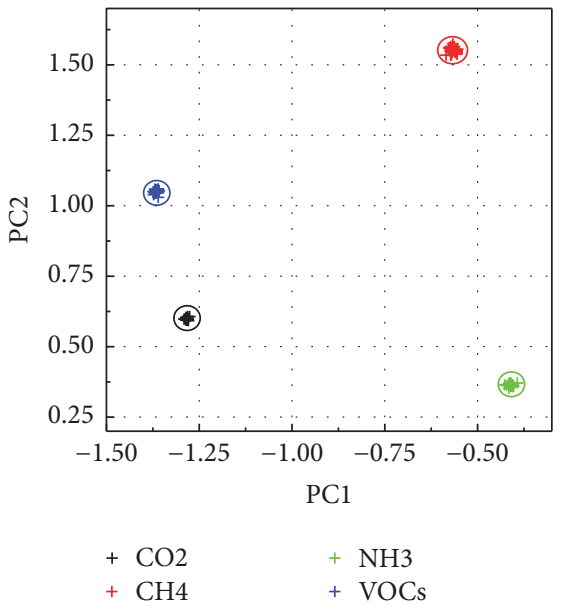

(c)

Figure 5: Classification results of SLLE when (a) $K=10$, (b) $K=20$, and (c) $K=30$. The horizontal axis represents the feature vector space corresponding to the selected first eigenvalue, and the vertical axis represents the selected second eigenvalue corresponding to the feature space. Both constitute a dimensionality reduction in two-dimensional space.

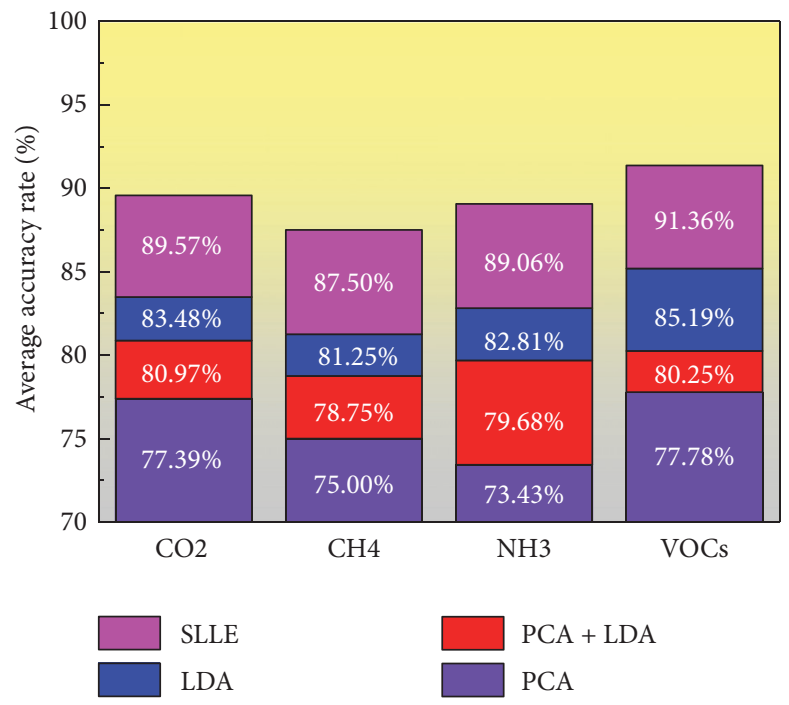

FIGURE 6: The results of the intuitive contrast average accuracy for the four sample gases. The horizontal axis represents the four sample gases, the vertical axis represents the accuracy of the recognition, and the four different colors represent the different algorithm results.

TABLE 3: The results of identification of the gas samples.

\begin{tabular}{lccccccc}
\hline \multirow{2}{*}{ Number } & \multirow{2}{*}{ Samples gas } & \multicolumn{3}{c}{ Data sets } & \multicolumn{3}{c}{ Average recognition count } \\
& & Train & Test & Total & PCA & PCA + LDA & LDA \\
\hline$(1)$ & $\mathrm{CO}_{2}$ & 168 & 115 & 283 & 89 & 93 & 96 \\
$(2)$ & $\mathrm{CH}_{4}$ & 124 & 80 & 204 & 60 & 63 & 65 \\
$(3)$ & $\mathrm{NH}_{3}$ & 95 & 64 & 159 & 43 & 51 & 70 \\
$(4)$ & VOCs & 84 & 81 & 135 & 63 & 65 & 53 \\
\hline
\end{tabular}




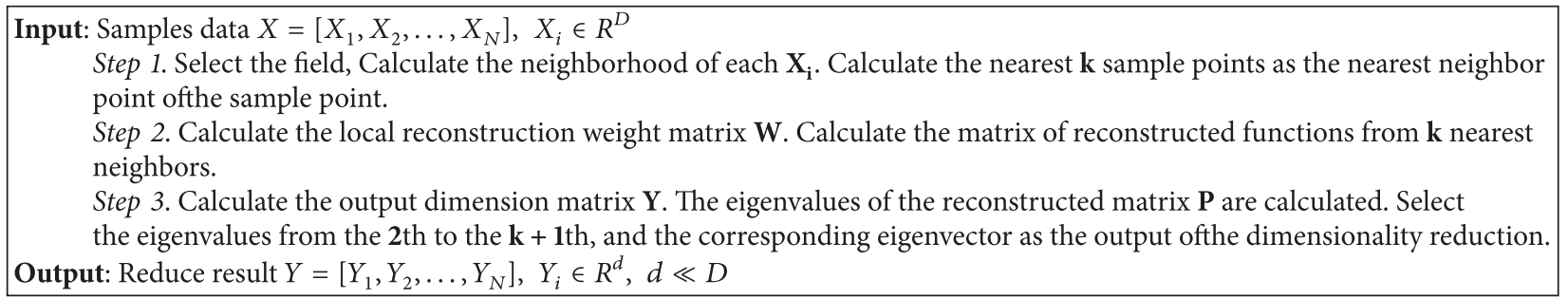

FIgURE 7: SLLE algorithm.

and the LDA and PCA + LDA tests accuracy rate is followed. PCA is used to test the accuracy rate of no more than $78 \%$. Therefore, the SLLE algorithm proposed in this paper is best used in the four algorithms when used to test the four kinds of sample gas data.

\section{Conclusion}

In this paper, we presented the proposed selective local linear embedding (SLLE) algorithm to reduce the dimension of collected gas data for industrial gas samples identification. The extracted high-dimensional data can maintain its original topology and have larger interclass spacing as well as smaller intraclass spacing. Compared to the traditional algorithms such as PCA and LDA, the SLLE performs better in distinguishing between different gases.

Therefore, SLLE algorithm with a low degree of freedom and simple procedure can enhance the accuracy of the selected gas samples detection, which endows higher sensitivity to olfactory machine. The SLLE algorithm is an effective method to quantitatively analyze various mixed gases, and it can meet the requirements of multimode gas-state identification. The outcome of the proposed approach can add more value to applications in the field of industrial gas monitoring and safety.

\section{Conflicts of Interest}

The authors declare that they have no conflicts of interest.

\section{Acknowledgments}

This work was supported by the National Natural Science Foundation of China (Grant no. 61571140), Guangdong Provincial Science and Technology Foundation of China (Grant nos. 2016B030303011, 2017A010101032), Guangdong Provincial Natural Science Foundation (Grant no. 2017A030310071), and Guangzhou Science and Technology Foundation of Guangdong Province (Grant no. 201607010247).

\section{Supplementary Materials}

Two tables and three figures covering more information on the following subjects are included: the detailed information of the employed PEN3 electronic nose and its technical specifications; full results for low concentration of $10 \mathrm{ppm}$ and $30 \mathrm{ppm}$, for the selected gases; and classification results for low gas concentration based on Euclidean distance (ED). (Supplementary Materials)

\section{References}

[1] J. Gutiérrez and M. C. Horrillo, "Advances in artificial olfaction: Sensors and applications," Talanta, vol. 124, pp. 95-105, 2014.

[2] A. Loutfi, S. Coradeschi, G. K. Mani, P. Shankar, and J. B. B. Rayappan, "Electronic noses for food quality: a review," Journal of Food Engineering, vol. 144, pp. 103-111, 2015.

[3] P. Batog and A. Wołczowski, "Sensor system for dynamic detection of the concentration gradient of volatile compounds in the air," in Proceedings of the 28th European Conference on Solid-State Transducers, EUROSENSORS 2014, pp. 1334-1337, September 2014.

[4] A. Vergara, E. Llobet, E. Martinelli, C. Di Natale, A. D’Amico, and X. Correig, "Feature extraction of metal oxide gas sensors using dynamic moments," Sensors and Actuators B: Chemical, vol. 122, no. 1, pp. 219-226, 2007.

[5] S. Marco, A. Gutiérrez-Gálvez, A. Lansner et al., "A biomimetic approach to machine olfaction, featuring a very large-scale chemical sensor array and embedded neuro-bio-inspired computation," Microsystem Technologies, vol. 20, no. 4-5, pp. 729742, 2014.

[6] L. Xu, J. He, S. Duan, X. Wu, and Q. Wang, "Comparison of machine learning algorithms for concentration detection and prediction of formaldehyde based on electronic nose," Sensor Review, vol. 36, no. 2, pp. 207-216, 2016.

[7] E. Phaisangittisagul and H. T. Nagle, "Predicting odor mixture's responses on machine olfaction sensors," Sensors and Actuators B: Chemical, vol. 155, no. 2, pp. 473-482, 2011.

[8] S. Zheng, W. Ren, and L. Huang, "Geoherbalism evaluation of Radix Angelica sinensis based on electronic nose," Journal of Pharmaceutical and Biomedical Analysis, vol. 105, pp. 101-106, 2015.

[9] D. L. García-González, N. Tena, R. Aparicio-Ruiz, and R. Aparicio, "Sensor responses to fat food aroma: A comprehensive study of dry-cured ham typicality," Talanta, vol. 120, pp. $342-$ 348, 2014.

[10] S. T. P. Radi, S. Ciptohadijoyo, W. S. Litananda, M. Rivai, and M. H. Purnomo, "Electronic nose based on partition column integrated with gas sensor for fruit identification and classification," Computers and Electronics in Agriculture, vol. 121, pp. 429435, 2016.

[11] F. Hossein-Babaei and A. Hooshyar Zare, "The selective flow of volatile organic compounds in conductive polymer-coated microchannels," Scientific Reports, vol. 7, Article ID 42299, 2017. 
[12] F. Hossein-Babaei and A. Amini, "Recognition of complex odors with a single generic tin oxide gas sensor," Sensors and Actuators B: Chemical, vol. 194, pp. 156-163, 2014.

[13] X.-M. Jia, Q.-H. Meng, Y.-Q. Jing, P.-F. Qi, M. Zeng, and S.G. Ma, "A new method combining KECA-LDA with ELM for Classification of chinese liquors using electronic nose," IEEE Sensors Journal, vol. 16, no. 22, pp. 8010-8017, 2016.

[14] S. Qiu and J. Wang, "Application of Sensory Evaluation, HSSPME GC-MS, E-Nose, and E-Tongue for Quality Detection in Citrus Fruits," Journal of Food Science, vol. 80, no. 10, pp. S2296S2304, 2015.

[15] D. Luo, H. G. Hosseini, and J. R. Stewart, "Application of ANN with extracted parameters from an electronic nose in cigarette brand identification," Sensors and Actuators B: Chemical, vol. 99, no. 2-3, pp. 253-257, 2004.

[16] S. T. Sarkar, A. P. Bhondekar, M. Macaš et al., "Towards biological plausibility of electronic noses: A spiking neural network based approach for tea odour classification," Neural Networks, vol. 71, pp. 142-149, 2015.

[17] D. H. Chen, S. B. Ye, X. H. Weng, J. Tong, and Z. Y. Chang, "Artificial olfactory system technology on chicken freshness detection," Applied Mechanics and Materials, vol. 461, pp. 801808, 2014.

[18] X. R. Wang, J. T. Lizier, A. Z. Berna, F. G. Bravo, and S. C. Trowell, "Human breath-print identification by E-nose, using information-theoretic feature selection prior to classification," Sensors and Actuators B: Chemical, vol. 217, pp. 165-174, 2015.

[19] D. Li, T. Lei, S. Zhang, X. Shao, and C. Xie, "A novel headspace integrated E-nose and its application in discrimination of Chinese medical herbs," Sensors and Actuators B: Chemical, vol. 221, pp. 556-563, 2015.

[20] J. Zhang, D. Gong, and Y. Zhang, "A niching PSO-based multirobot cooperation method for localizing odor sources," Neurocomputing, vol. 123, pp. 308-317, 2014. 


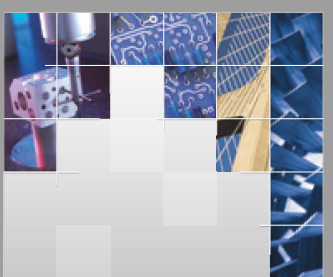

\section{Enfincering}
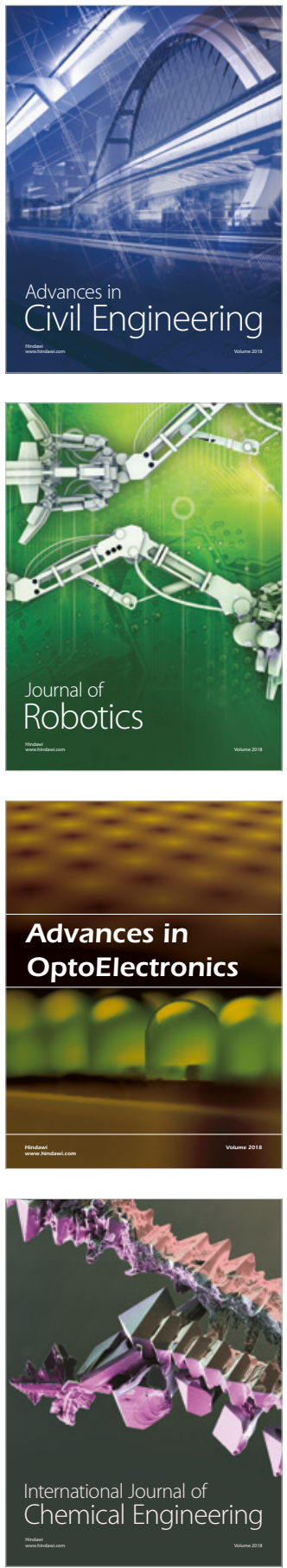

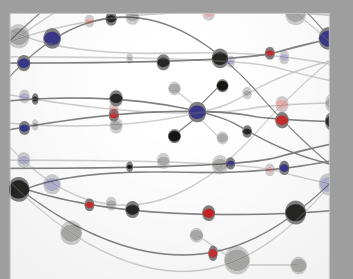

\section{Rotating \\ Machinery}

The Scientific World Journal

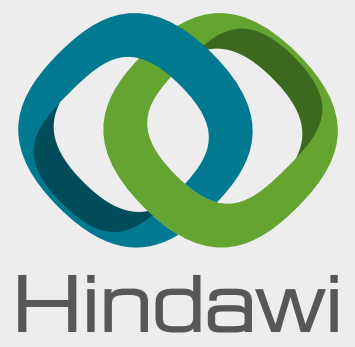

Submit your manuscripts at

www.hindawi.com
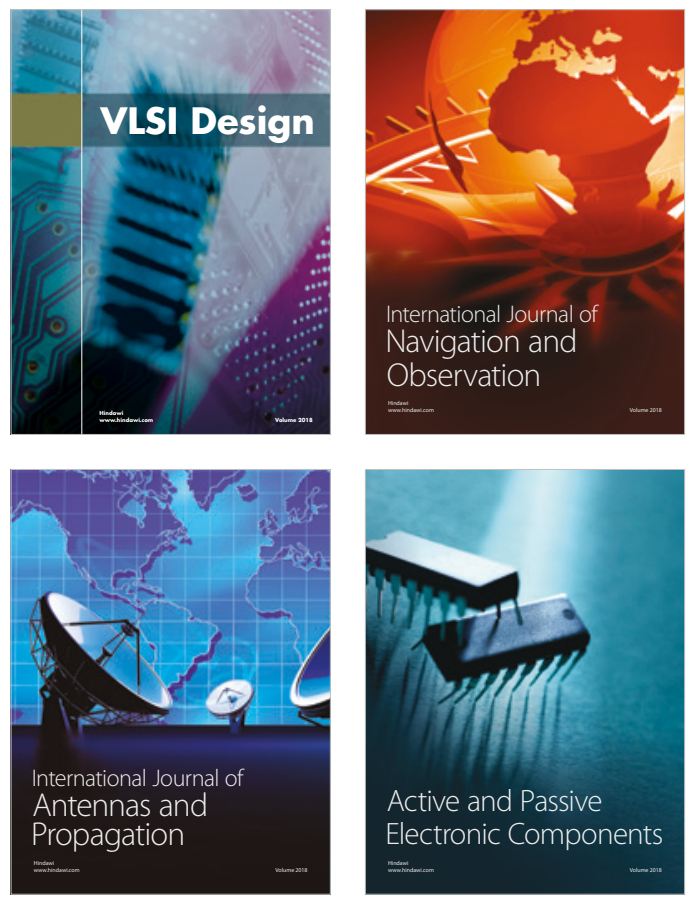
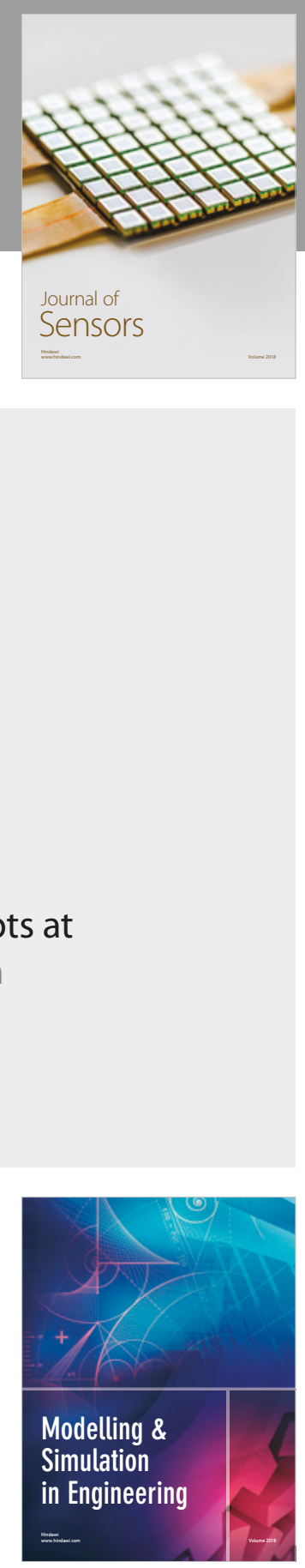

\section{Advances \\ Multimedia}
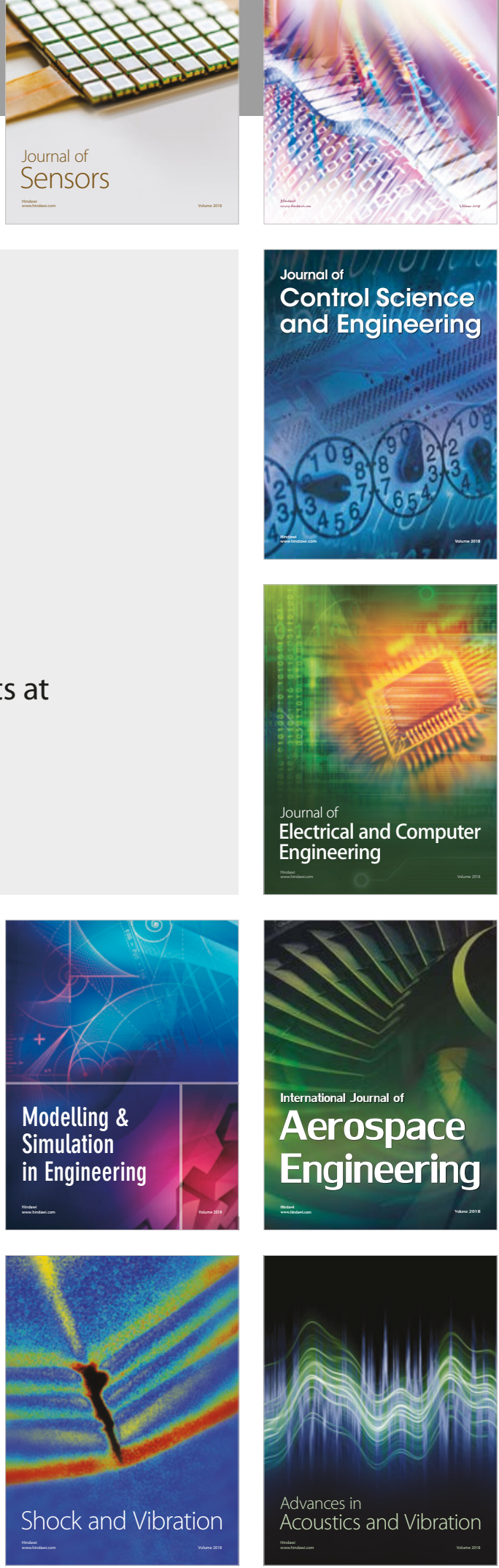